\title{
DARK MATTER IN CLUSTERS OF GALAXIES
}

\author{
CLAUDE R. CANIZARES \\ Department of Physics and Center for Space Research \\ Massachusetts Institute of Technology \\ Cambridge, MA 02146, USA \\ (crc@mit.edu)
}

An appropriate subtitle for this talk might be "Newton meets Einstein." For many decades, the prime tool for studying the amount and distribution of matter in galaxy clusters was decidedly Newtonian, involving at first the measurements of the dynamics of the galaxies themselves and, for the past 15 years or so, the imputed dynamics of the hot, X-ray emitting intra-cluster gas. Einstein enters more recently with the introduction of gravitational lensing as a tool for studying cluster mass distributions. Rapid progress is being made in each of these areas, and there are now attempts to bring them together to give a consistent and more accurate picture of clusters.

\section{Newton's Tools}

Dynamical measures of the quantity of mass in clusters rely on the basic Newtonian relation $M(<r) \approx v^{2} r / G$, with the exact relation depending on the assumptions about the test particles, whether galaxies or electrons. For galaxies, the relevant velocity is the line of sight velocity dispersion, which has typical values around $10^{3} \mathrm{kms}^{-1}$. One assumes that the system is virialized or relaxed and incorporates various possibilities for likely anisotropies in the galaxy orbits. This method is fundamentally limited by the statistics of the finite number of galaxies as well as possible ambiguities about cluster membership.

In the case of X-ray observations, the test particles are electrons in the diffuse, hot plasma whose velocity is determined by measuring the plasma temperature, typically $10^{8} \mathrm{~K}$. Here one is not troubled by velocity anisotropies, because the plasma is a collisional gas, but it is necessary to assume hydrostatic equilibrium, an assumption made plausible by the very 
regular, nearly circular X-ray images of many clusters (Forman and Jones, 1982; Jones and Forman, 1984).

Uncertainties about the galaxy orbits or temperature gradients typically introduce uncertainties of order a factor of 2 or so in the mass within some radius, although as I will show below, the new X-ray temperature measurements are now yielding mass measurements good to 10 or $20 \%$ in some clusters.

It is worth remembering that given the typical velocities in clusters, one can move no more than $10 h_{100}^{-1} \mathrm{Mpc}$ in a Hubble time (where $H_{o}=100 h_{100}$ $\mathrm{km} \mathrm{s}^{-1} \mathrm{Mpc}^{-1}$ ). Thus all dynamical methods should only be applied with confidence within, say, several Mpc.

While clusters have been studied, there are relatively few for which a full, joint analysis of optical and X-ray data has been performed. One example is the righ cluster A2256 studied by Henry et al. (1993) and Fabricant et $a l$. (1989) with the ROSAT observatory. The derived total mass density for A2256, and the visible components in gas or galaxies, are shown in Fig. 1. As is generally the case, the analysis gives a rather good determination of mass within $0.5-1 \mathrm{Mpc}$, where the X-ray measurements are best. Extrapolations to larger radii are interesting but necessarily less accurate. Similarly, on smaller scales the data are less constraining. In many clusters, this is also the region where $\mathrm{X}$-ray data are complicated by the presence of cooling flows (see below). This is relevant when we compare to some of the gravitational lensing results. Another feature of Fig. 1 which is common to most clusters is that the mass in gas exceeds the mass in galaxies. But for an accident of history, these systems might not be called "clusters of galaxies" at all. For A2256, Henry et al. find a total binding mass of $0.5 \pm 0.1 \times 10^{15} \mathrm{M}_{\odot}$ within $0.76 h_{100}^{2} \mathrm{Mpc}$.

In addition to radial distribution, there are a few cases for which we have attempted to measure the degree of flattening of the dark matter. My student, David Buote, and I studied the cores of five rich Abell clusters in which the galaxy distribution appears highly flattened on the sky (ellipticity $\approx 0.5$; see Buote and Canizares, 1992). In all five clusters, we find that within $\approx 0.5 \mathrm{Mpc}$ the X-ray contours are indeed elongated and agree with the galaxies in position angle, but with substantially smaller ellipticity. We conclude that the dark matter must also be less elongated than the galaxies, with ellipticity $\approx 0.2$. Following Plionis et al. (1991), we argue that these findings may be generally applicable to the majority of clusters.

One interesting newer result is the evidence for significant quantities of dark matter in poor groups (Mulchaey et al., 1993; Ponman and Bertram, 1993; Ponman et al., 1994; Henriksen and Mammon, 1994; David et al., 1994). The ROSAT instrument is very well suited for detecting these systems. For example, David et al. (1994) showed that, outside the cooling 


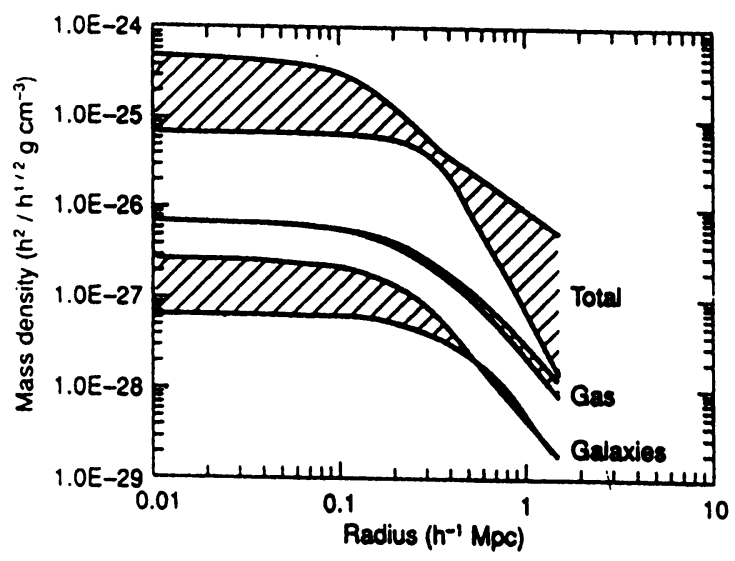

Figure 1. The total, hot gas and galaxy mass densities as a function of radius in A2256 as determined from the X-ray analysis of Hentry et al. (1993). The width of the band gives an estimate of the $68 \%$ confidence ranges.

flow in the core, the NGC 5044 group is nearly isothermal to at least 250 kpc. They find a total mass of $110^{13} h_{100}^{-1} \mathrm{M}_{\odot}$ and $M / L \approx 200 h_{100}$, which are typical of these systems.

The ASCA satellite (Tanaka et al., 1994) carries an imaging CCD with much improved spectral resolution and a wider bandpass more suited to the higher temperatures of rich clusters. It's angular resolution is 2 arc min, worse than that of ROSAT but not debilitating for the study of clusters. Results are just now beginning to emerge for many of the well known clusters (cf. Mushotzky, 1995). ASCA generally confirms that clusters are nearly isothermal outside the very central cooling flow regions, although there are clear indications for some temperature and abundance variations that may reflect spatial subclustering (Arnaud et al., 1994; Fukuzawa et al., 1994 ).

To summarize, there is abundant evidence from dynamical studies that clusters with a wide range of richness contain significant amounts of dark matter. The X-ray data have traditionally been fit by models with a core radius, within which the density becomes constant; with $\rho(r) \propto r^{-2}$ and $\propto r^{-3}$ at larger radii. Typical core radii are 100 to $400 \mathrm{kpc}$. The total cluster mass within, say, $0.8 \mathrm{Mpc}$ is typically $510^{14} h_{100}^{-1} \mathrm{M}_{\odot}$; beyond this radius the estimates are increasingly uncertain, although one would expect that total masses of rich clusters exceed $10^{15} \mathrm{M}_{\odot}$. The derived ratios of mass to (blue) light range from 150 to 300 . The critical assumption of isothermality of X-ray gas, long a weak point of mass determinations, is being confirmed by recent observations. Both this and the assumption of hydrostatic equilibrium are also reasonably consistent with the elaborate 

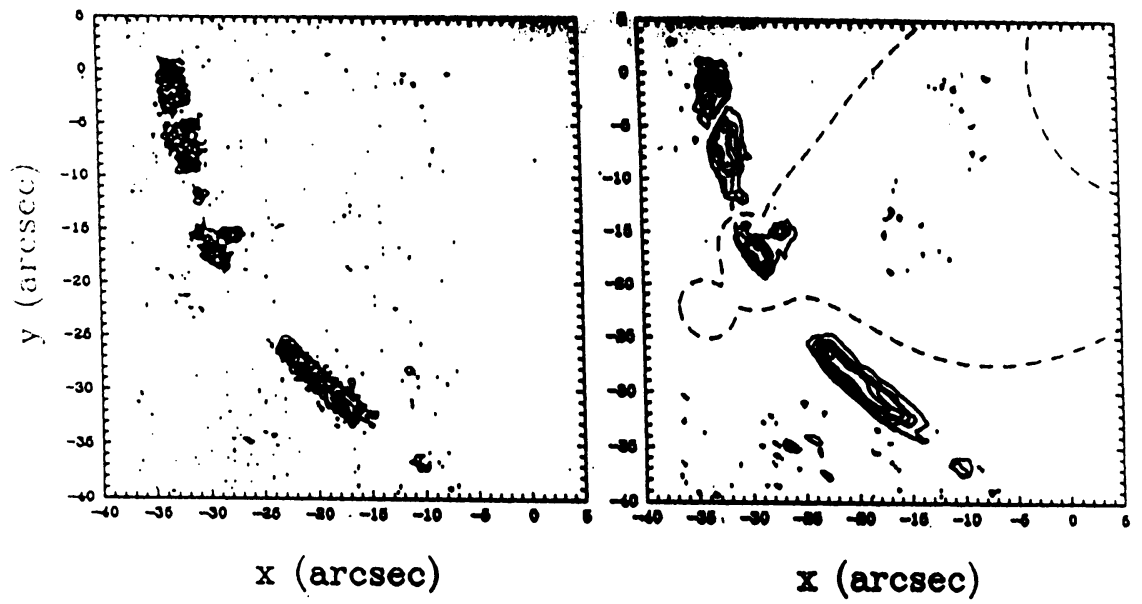

Figure 2. Image of the giant arc in Cl0024+1654 (left) and the best model reconstruction of the are (right) from Wallington et al. (1994). The model includes a smooth cluster mass plus mass associated with individual cluster galaxies.

$\mathrm{N}$-body plus hydrodynamic models of the inner regions of well evolved clusters now being run by several groups (e.g. Tsai et al., 1994; Evrard, 1995). One example of the observed and reconstructed image shown in Fig. 2 ; the correspondence is remarkable.

In the centers of many, probably most, clusters, X-ray observations show significant quantities of relatively cool gas, indicating the presence of a "cooling flow" (Fabian, 1994; Fabian et al., 1991). ASCA, with its much improved spectral resolution, is confirming the earlier Einstein and ROSAT findings (Fabian et al., 1994), which suggest that several hundred solar masses per year are cooling below $310^{6} \mathrm{~K}$ and disappearing from the X-ray phase. Over the lifetime of a cluster, this is a sizable amount of material, comparable to a giant galaxy. The detection of excess X-ray absorption in the centers of some clusters indicates that at least some of the matter may remain in the form of dark cold clouds, while the rest might form predominately low mass stars In any event, this dark matter could be an important contribution to the central $\approx 100 \mathrm{kpc}$ of clusters and the dominant galaxy that often resides there, is only a minor fraction of the total cluster dark matter. It should be noted at a meeting on stellar populations, however, that cooling flows may well be the places where we can observe at close hand a process related to galaxy formation at earlier epochs.

\section{Einstein's Tools}

The study of clusters using gravitational lenses is growing very rapidly at present (see reviews by Soucail, 1991; Schneider et al., 1992; Kayser et al., 1992; Fort and Mellier, 1994; and references therein). Clusters, and the 
galaxies they contain, are very effective gravitational lenses, which both magnify and distort the images of distant galaxies seen through the cluster. The strongest portion of the lens is generally within the central 10-20 arc sec of the cluster, but weak lensing extends to much larger distances. Within the central region, one often sees very elongated arcs, the highly distorted images of a galaxy at much higher redshift. In the weaker lensing region, galaxies are only slightly distorted, into so-called "arclets." Individually, these are too small and faint to be studied in detail, but one can treat these statistically and thereby map the projected gravitational potential. The lensing responsible for multiply imaged quasars is dominated by galaxy-sized masses making it more difficult, though not impossible, to infer information on cluster mass (e.g. Falco et al., 1991).

In the "thin lens" approximation appropriate for clusters, the strength of the lens is related to the surface mass density projected on the sky. To get strong lensing, required for the so-called giant arcs, the mass density must be $\geq \Sigma_{\text {crit }}$, which has the surprisingly terrestrial value of $\approx 0.5-$ $1.0 \mathrm{gm} \mathrm{cm}^{-2}$. For reference, this is comparable to a few times the closure density (corresponding to $\Omega=1$ over the Hubble distance $D_{H_{o}}=c / H_{o}$ ) Expressed in terms of mass enclosed within the radius of the arc, one has

$$
M\left(R_{a r c}\right) \approx \frac{c^{2} R_{a r c}}{4 G}\left(\frac{R_{a r c}}{D_{H_{o}}}\right)\left(\frac{D_{O S}}{D_{O L} D_{L S}}\right) .
$$

Note that this is similar to the dynamical equation of $\S 1$, with $v^{2}$ replaced by $c^{2}$. The extra factor $r_{a r c} / D_{H_{o}}=10^{-5}\left(r_{a r c} / 30 \mathrm{kpc}\right) h_{100}$ compensates for the large value of the velocity of light. The last factor, the ratio of angular diameter distances from observer to source, observer to lens, and lens to source, is of order unity.

Fort and Mellier (1994) catalog approximately three dozen clusters in which arcs or arclets have been detected. The clusters range in redshift from 0.15 to 0.5 , in other words not too close and not too far, but just right for lensing a background object. Roughly half these show giant arcs, many of which have measured redshifts of 0.5-2.2. It is relevant for a meeting on stellar populations to note that these gravitational lenses are natural telescopes for the study of the stellar content of galaxies at very early epochs in the universe.

The typical procedure for deducing the mass of a cluster from the image of the arc involves iterative modeling of the mass distribution, usually beginning with a simple, ellipsoidal mass distribution and progressively adding higher order effects, such as those due to individual cluster galaxies (e.g. see Wallington et al., 1994).

Although relatively few arc systems have been modeled in detail, we can summarize the current findings. First, there is at least qualitative a- 
greement between the lensing models and the dynamical results described above, both optical and X-ray. Given the complete independence of these methods, this agreement is very comforting. As to the more detailed shape of the mass, for two cases (MS2137-23 [Mellier et al., 1993; Miralda-Escude, 1994] and A370 [Kneib et al., 1993]) the analysis indicates that mass can follow the light distribution of the elliptical $\mathrm{cD}$ galaxies (two of them for A370) in the central regions of the clusters. On the other hand, the detailed characteristics of the arcs (specifically, the ratio of length to width, which depends on the gradient of the surface potential) and the high incidence of arcs (Grossman and Saha, 1994; Fort and Mellier, 1994; and references therein) imply smaller core radii and considerably higher central surface mass densities than were imputed from the X-ray data. Much has been made of this discrepancy over the past few months (e.g. Miralda-Escude and Babul, 1994), including claims that total cluster masses may have previously been underestimated by factors of three or more. I (and others) suspect that most or all the discrepancy results from the limitations of the models being employed (e.g. see Loewenstein, 1994; Bartelmann and Weiss, 1994). Models with a single core radius, which when normalized in the central region to explain the arcs and then extrapolated by an order of magnitude or more in radius, exceed the mass determined by the X-rays. But the X-ray observations generally do not constrain the central regions very well, as we have noted above, and are often further confused by of the presence of cooling flows. It seems likely that alternative models can accommodate X-ray and lensing abservations.

We also need to recall that lensing is a measure of a two dimensional surface potential, while the X-rays measure the three-dimensional potential. The total mass along the line of sight required to form a typical arc is several times $10^{13} \mathrm{M}_{\odot}$, which is the mass of a small group. Thus projection effects, substructure, etc., can be significant for lensing. We need a larger sample to sort this out (see also below).

The study of arclets is at a still earlier stage of development, but it has tremendous promise and is moving rapidly. Following the pioneering work of Tyson et al. (1990), statistical studies of the distribution of faint galaxy distortions are being performed on an increasing number of clusters (e.g. Bonnet et al., 1994; Smail et al., 1994; Fahlman et al., 1994). Kaiser and Squires (1993) have developed an elegant inversion technique for reconstructing the surface mass density from a map of the distortions. Since the distortions are caused by gradients in the surface potential, the reconstructed surface mass density is a lower limit. Although only a few clusters have been analyzed so far, this method will eventually give the best measure of masses $>10^{15} \mathrm{M}_{\odot}$ on scales $>1 \mathrm{Mpc}$.

On the other hand, both the power and the weakness of arclet inversion 
is that it probes the total mass along the line of sight, not just that concentrated in a cluster. This raises the exciting possibility of studying mass structures outside the relatively rare rich clusters. But the application to clusters suffers because of the susceptibility to projection effects; since we know from large scale structure studies that clusters generally lie at the intersections of filaments and sheets of galaxies (and presumably mass), one must be cautious in drawing conclusions about the three dimensional potential from studies of its projection. In fact, some of the first results of arclet inversions do imply masses well in excess of those determined by dynamical means, and even suggest global densities well above closure (i.e. $\Omega \approx 2$; e.g. Fahlman et al., 1994).

\section{The Baryon Problem}

In the context of modern cosmology, the ample evidence for dark matter in clusters, once considered remarkable, is entirely expected. In fact, the current puzzle is why there is not more of it. Over the past several years, $\mathrm{X}$-ray analyses have shown that the total baryon content of clusters, stars plus hot gas, is around $4-7 h_{100}^{-3 / 2} \%$ (e.g. Briel et al., 1992; White et al., 1993; White and Fabian, 1994). This assumes, of course, that none of the dark matter is baryonic, so it is really a lower limit. However, standard big bang nucleosynthesis sets an upper limit to the baryon fraction of the universe of around $1.25 \Omega_{o} h_{100}^{-2} \%$ (e.g. Copi et al., 1994). Because clusters constitute a small fraction of the total mass of the universe, this would generally not be considered a serious discrepancy even for $\Omega=1$. But recently White et al.(1994) showed that one cannot have large enhancements of baryons in clusters relative to the cosmic mean (on the contrary, difficiencies are expected from N-body simulations; Kang et al., 1994). They concluded that the high baryon fraction in clusters is "a challenge to cosmological orthodoxy," in which $\Omega=1$ as a result of the inflationary scenario. Since this was written by some of the high priests of cosmological orthodoxy, it must be taken very seriously. Some would argue for a subcritical universe, with $\Omega<0.2$, but there is at least some evidence for larger $\Omega$ from studies of large scale streaming motions of galaxies (e.g. Dekel et al., 1993). However this issue is resolved, it is likely to reveal something interesting about the universe.

\section{Conclusion}

There is now ample evidence that $60-80 \%$ of the matter in clusters, rich and poor, and probably also in groups of galaxies is dark. Both the visible galaxies and the X-ray emitting gas correlate with the dark matter, but 
neither is an exact tracer. Cluster masses are generally $\approx 510^{14} \mathrm{M}_{\odot}$ within $\approx 0.8 \mathrm{Mpc}$ implying that $\mathrm{M} / \mathrm{L}$ is several hundred. The newer gravitational lens maps do not always agree in detail with dynamical mass estimates, although this is very much "work in progress" which is likely to significantly improve our understanding of the mass distribution in clusters. The studies of arclets may allow us to map mass distributions at still larger radii, and even outside of clusters altogether. Finally, the large baryon fractions found in clusters and groups stands as a major puzzle for standard cosmology.

It is a pleasure to thank David Buote, Eric Gaidos, and John Tsai for useful conversations during the preparation of this talk.

\section{References}

Arnaud, J. et al., $1994 A p . J .$, in press

Bartelmann, M. and Weiss, A., $1994 A$. $\& A .287,1$

Bonnet, H., Mellier, Y. and Fort, B., 1994 Ap. J.427, L83

Buote, D. and Canizares, C., 1992 Ap. J.400, 385

Copi, C., Schramm, D. and Turner, M., 1994, Science, in press

David, L. et al., 1994, Ap. J., in press

Dekel, A. et al., 1993, Ap. J.412,1

Durret et al., eds., 1995, Clusters of Galaxies, Editions Frontieres, in press

Evrard, G., 1995, in Durret et al. (op cit)

Fabian, A. C., 1984, Ann. Rev. Astron. Astrophys.32, 277

Fabian, A. C., ed., 1991, Clusters and Superclusters of Galaxies, Kluwer

Fabian, A. C. et al., 1994, Ap. J., in press

Fabian, A. C., Nulsen, P. and Canizares, C., 1991, A. \& A.Rev. 2, 191

Fabricant, D., Kent, S. and Kurtz, M., 1989, Ap. J.336, 77

Fahlman et al., 1994, preprint

Falco, E., Gorenstein, M. and Shapiro, I., 1991, Ap. J.372, 364

Forman, W. and Jones, C., 1982, Ann. Rev. Astron. Astrophys.20, 547

Fort, B. and Mellier, Y., 1994, A. \& A.Rev. 5, 239

Fukuzawa Y. et al., 1994, Pub. Astr. Soc. Japan46, L141

Jones, C. and Forman, W., 1984, Ap. J.276, 38

Kang, H. et al., 1994, Ap. J.428, 1

Kayser, R., Schramm, T. and Nieser, L., eds., 1992, Gravitational Lenses, Springer-Verlag

Henriksen, M. and Mamon, G., 1994, Ap. J.421, L63

Henry, P., Briel, U. and Nulsen, P., 1993, A. \& $A .271,413$

Kaiser, N. and Squires, G., 1993, Ap. J.404, 441

Kneib, J.-P. et al., 1993, A. \& A.273, 367

Loewenstein, M., 1994, Ap. J.431, 91

Mellier, Y., Fort, B. and Kneib, J.-P., 1993, Ap. J.407, 33

Miralda-Escude J., 1994, preprint

Miralda-Escude J. and Babul, 1994, preprint

Mulchaey et al., 1993, Ap. J.404, L9

Mushotzky, R., 1995, in Durret et al. (op cit)

Plionis, M. Barrow, J. D. and Frenk, C. S., 1991, M. N. R. A. S.249, 662

Ponman, T. and Bertram, D., 1993, Nature 363, 51

Ponman, T. et al., 1994, Nature 369, 462

Sarazin, C., 1988, X-ray emission from Clusters of Galaxies, Cambridge U. Press

Schneider, P., Ehlers, J. and Falco, E., 1992, Gravitational Lenses, Springer-Verlag

Smail et al., 1994, M. N.R. A. S., in press 
Soucail, G. 1991, in Fabian (op cit)

Tsai, J., Katz, N. and Bertschinger, E., 1994, Ap. J.423, 553

Tanaka, Y., Inoue, H. and Holt, S., 1994, Pub. Astr. Soc. Japan46, L37

Tyson, A., Valdes, F. and Wenk, R., 1990, Ap. J.349, L1

Wallington, S., Kochanek, C. and Koo, W., 1994, Ap. J., in press

White, D.A. and Fabian, A.C., 1994, M. N.R.A.S., in press

White, S.D.M. et al., 1993, Nature 366, 429

G. BURBIDGE: Two points: (1) It is generally supposed that the dark matter mass can be estimated by assuming that the clusters or groups are stable so that the virial theorem holds. While this is true for many clusters there are others in which there is no evicdence for stability. Abartsumian said that thee were systems of positive energy, i.e., coming apart. This view should still be carefully examined and not ignored. (2) A good alternative to the standard big bang theory has been developed by Hoyle, Burbidge and Narlikar (papers in Ap.J., M. N. R.A.S. and $A$. $\mathscr{E} A$. 1993, 1994). In that theory, all of the dark matter is baryonic and there is no non-baryonic matter. Thus we predict that the bulk of the mass is baryonic and is many times the luminous matter.

CANIZARES: I agree that everything I have said is in the context of the standard big bang cosmology. In particular, it is clear that the baryon fraction is only a problem in that context. Even in big bang cosmology, it is not a problem if you allow $\Omega<1$.

DE ZEEUW: (1) Your "worries" $\sum(r)$ and $\rho(r)$ not matching surprised me. I think it is wonderful to have a method that measures mass even if there are no luminous tracers, such as galaxies. (2) Why do you say that observers want $\Omega=1$ ? Isn't this theoretical prejudice?

CANIZARES: I was only saying that the maps of surface mass density need to be interpreted cautiously when drawing conclusions about the three dimensional mass distribution in a given cluster. But this "weakness," if I can call it that, is also possibly an even greater "strength" in the more general question of tracing mass independent of whether or not it is in cluster. As to evidence that $\Omega=1$, the observational input comes from the studies of large-scale streaming velocities. As you know, this is not a strong constraint at present, but it does favor larger values of $\Omega$

MEWE: You mentioned that the ASCA observations confirm the presence of cooling flows. However from the mapping you can not draw such a conclusion because the image spatial resolution of 3 arcmin is too curde. I think you mean that the indirect evidence comes from the x-ray spectral fitting where you need an extra cool component for a better fit?

CANIZARES: Yes, that is just what I meant. 